\title{
Genetic Vasopressin Deficiency Facilitates Performance of a Lateralized Reaction-Time Task: Altered Attention and Motor Processes
}

\author{
J. David Jentsch \\ Department of Psychology, University of California, Los Angeles, California 90095-1563
}

\begin{abstract}
Brattleboro rats are a variety of the outbred Long-Evans strain that possess a single nucleotide deletion in the second exon of the arginine vasopressin gene, resulting in the synthesis of an altered protein that does not enter the normal secretory pathway. Rats heterozygous $(\mathrm{di} /+)$ for the deletion have a partial vasopressin deficiency and exhibit a variety of behavioral and neurochemical alterations compared with normal wild-type Long-Evans rats, which provide evidence for a CNS function for vasopressin. Here, we examined the acquisition and performance of a test of visuospatial attention by di/ + Brattleboro rats and their wild-type Long-Evans control counterparts. Surprisingly, di/ + rats exhibited superior performance of the task compared with wild-type controls; performance differences included greater accuracy of detection of visual target stimuli, faster overall reaction times, and fewer trial omissions. Di/ + rats also exhibited more approaches to the reinforcer receptacle at nonreinforcement times. These results indicate that alterations of vasopressin signaling result in a clear cognitive phenotype, including faster motor initiations and superior choice accuracy in a test of visual attention.
\end{abstract}

Key words: attention; vasopressin; Brattleboro rat; cognition; autism; schizophrenia

\section{Introduction}

Although little is known about the biochemical pharmacology of vasopressinergic synapses in the brain, substantial evidence supports the notion that this nonapeptide plays a role in CNS function. Receptors for vasopressin (the V1A receptor in particular) are expressed in a number of forebrain structures (Barberis and Tribollet, 1996), and pharmacologic manipulations of the vasopressin system can produce alterations of cognitive and social behaviors in rats (Insel et al., 1999). Although the effects of vasopressin analogs on cognition are inconsistent, overall (Strupp and Levitsky, 1985), the evolving evidence seems to suggest that vasopressin regulates various aspects of cognition, including learning, memory, and/or attention.

Evidence for a role for vasopressin in cognition comes from studies of the Brattleboro rat. Brattleboro rats are a variety of the Long-Evans strain that possess a single nucleotide deletion in the second exon of the arginine vasopressin gene. The resulting frame shift causes synthesis of an altered protein that does not enter the normal secretory pathway. Rats homozygous (di/di) for the deletion have no detectable circulating vasopressin concentration, exhibit a variety of behavioral and neurochemical alterations compared with normal wild-type Long-Evans rats, and have diabetes insipidus. Heterozygous $(\mathrm{di} /+)$ Brattleboro rats are partially vasopressin-deficient. A number of studies have indicated that di/di Brattleboro rats exhibit deficits of social recognition (i.e., affiliative behavior) (Engelmann and Landgraf, 1994), conditioned fear (i.e., affective learning) (Stoehr et al., 1993), delayed

\footnotetext{
Received Sept. 3, 2002; revised 0ct. 29, 2002; accepted Nov. 13, 2002.

This work was supported by the University of California. I thank Alexander Arguello for artistic contributions, J. Kristin Martin for technical assistance, and Luigi Anzivino for useful discussions.

Correspondence should be addressed to Dr. J. David Jentsch, Department of Psychology, University of California at Los Angeles, P.0. Box 951563, Los Angeles, CA 90095-1563. E-mail: djentsch@ucla.edu.

Copyright $\odot 2003$ Society for Neuroscience $\quad 0270-6474 / 03 / 231066-06 \$ 15.00 / 0$
}

alternation (i.e., working memory) (Colombo et al., 1992), and prepulse inhibition (i.e., sensorimotor gating) (Feifel and Priebe, 2001), with di/ + rats generally showing a partial phenotype. It has been suggested that these data indicate that vasopressin plays a role in regulating a number of psychological domains.

The behavioral impairments in Brattleboro rats have been interpreted as evidence that these mutant rats may serve not only as a model for vasopressin deficiency but also for autism (Insel et al., 1999) and/or schizophrenia (Feifel and Priebe, 2001). Central to both of these disorders are alterations of visual attention; patients with schizophrenia exhibit deficits of sustained attention (Nuechterlein, 1977) as well as impairments of executive aspects of attention (e.g., attention shifting) (Pantelis et al., 1997). Interestingly, patients with autism show enhancements of low-level visual processing, including visual search, feature discrimination, and superior focused versus divided attention (Plaisted et al., 1999; O'Riordan and Plaisted, 2001; O'Riordan et al., 2001). These facilitations of low-level visual processing and attention appear to be in tandem with impairments of executive aspects of attention (Allen and Courchesne, 2001).

We sought to evaluate the acquisition and performance by $\mathrm{di} /+$ Brattleboro and wild-type rats of a lateralized reaction-time task that measures visuospatial attention and motor initiation; homozygous animals were not studied because of the concern that the consequent diabetes insipidus may complicate comparisons of food-motivated performance. In the task used, animals initiate the presentation of a visual target stimulus at one of two possible spatial locations and then make a response toward the location of the target. Therefore, this task requires sustained and divided attention. We also evaluated response times to gauge motor-initiation speeds in both groups. Here, we report the results of these investigations; partial vasopressin deficiency produces superior accuracy of detection and faster response times. 


\section{Materials and Methods}

Subjects. Sixty-day-old male rats [heterozygous $(\mathrm{di} /+)$ Brattleboro or wild-type Long-Evans rats] were purchased from Harlan (Indianapolis, IN) and were housed in genotype-specific triads on a 14.5/9.5 hr light/ dark cycle, with lights on at 6:30 A.M.; all behavioral testing was conducted during the light phase. The subjects were initially deprived of food to $90 \%$ of their free-feeding weights and subsequently fed $15 \mathrm{gm}$ of rat chow per day in their home cages ( $1 \mathrm{hr}$ after testing). Water was continuously available except during the testing period. This feeding schedule was sufficient to allow all subjects to gain $\sim 5-10 \%$ body weight per week. The experimental protocols used were consistent with the National Institutes of Health's Guide for the Care and Use of Laboratory Animals and were approved by the Chancellor's Animal Research Committee at the University of California at Los Angeles.

Behavioral testing apparatus. The design of the operant chambers is based on that described by Carli et al. (1983). Standard extra-tall aluminum and Plexiglas operant chambers equipped with a light bulb and infrared beam pellet-delivery magazine on one side and a curved panel with five nose-poke apertures on the opposite side (Med Associates, E. Fairfield, VT) were used. The boxes were housed inside of a soundattenuating cubicle; background white noise was broadcast, and the environment was illuminated with a house light (a light diffuser that was located outside of the operant chamber but within the cubicle).

Pretraining. All of the rats first received free reinforcer ( $45 \mathrm{mg}$ Bioserv dustless precision; Bio-Serv, Frenchtown, NJ) pellets in their home cages for 3 consecutive days before commencing training. Subsequently, all rats were trained in a single session in which the house light was continuously illuminated and single pellets were delivered into an illuminated magazine on a fixed-time $20 \mathrm{sec}$ schedule over a $45 \mathrm{~min}$ period.

One day after magazine training, the rats were trained to make a sustained nose-poke at the center aperture in three consecutive daily sessions. On the first day, the session began with illumination of the house light; a variable-duration nose-poke of $10,200,400$, or 600 msec was required in the illuminated center aperture to trigger a pellet to be dispensed within the head-entry magazine on the back wall (the nose-poke duration requirements were varied randomly from trial to trial). When the rat successfully responded for the duration of the hold period, the head-entry magazine was illuminated and a pellet was dispensed. After the rat retrieved the pellet, the magazine light was extinguished, and $3 \mathrm{sec}$ later the center aperture was illuminated to signal the initiation of another trial. The session terminated after $60 \mathrm{~min}$ passed or the rat earned 100 pellets, whichever occurred first. On the second and third days, the procedure was identical except that the rat was required to sustain 10 , 200,500 , or $700 \mathrm{msec}$ nose-pokes on the second day and 200, 500, 700, or $1000 \mathrm{msec}$ nose-pokes on the third day.

Acquisition of the lateralized reaction-time task. After being trained to make the sustained nose-poke, rats began daily testing on the lateralized reaction-time task (the general task contingency is shown in Fig. 1); in the first four sessions, a target stimulus of fixed duration was presented for all trials in a session (which terminated after $60 \mathrm{~min}$ or 128 trials, whichever came first). The task began with the illumination of the house light and the rats retrieving a single pellet from the magazine. The center aperture on the opposite wall was illuminated 3 sec later. The rat was then required to make a sustained, variable-duration nose-poke $(200,500,700$, or 1000 $\mathrm{msec}$ ) in the center aperture. After the sustained nose-poke was completed, the far left or far right aperture was illuminated for a fixed period $(30,5,2.5$, or $1 \mathrm{sec}$ on days $1,2,3$, or 4 of training, respectively). During target presentation, a nose-poke response at that aperture resulted in a pellet being delivered at the magazine, and a "correct" choice was scored. A limited hold period also applied on days 3 and 4; a response within $5 \mathrm{sec}$ of onset of target illumination was reinforced. Three seconds after the pellet was retrieved, the center aperture was illuminated to signal the onset of another trial.

When a rat responded at a location that was not that of the target during target presentation or within the limited hold period, all lights in the box were extinguished, and the rat was given a $3 \mathrm{sec}$ "time-out" period in complete darkness; in this case, an "incorrect choice" was scored. In addition, if the rat made no response within the target presen-
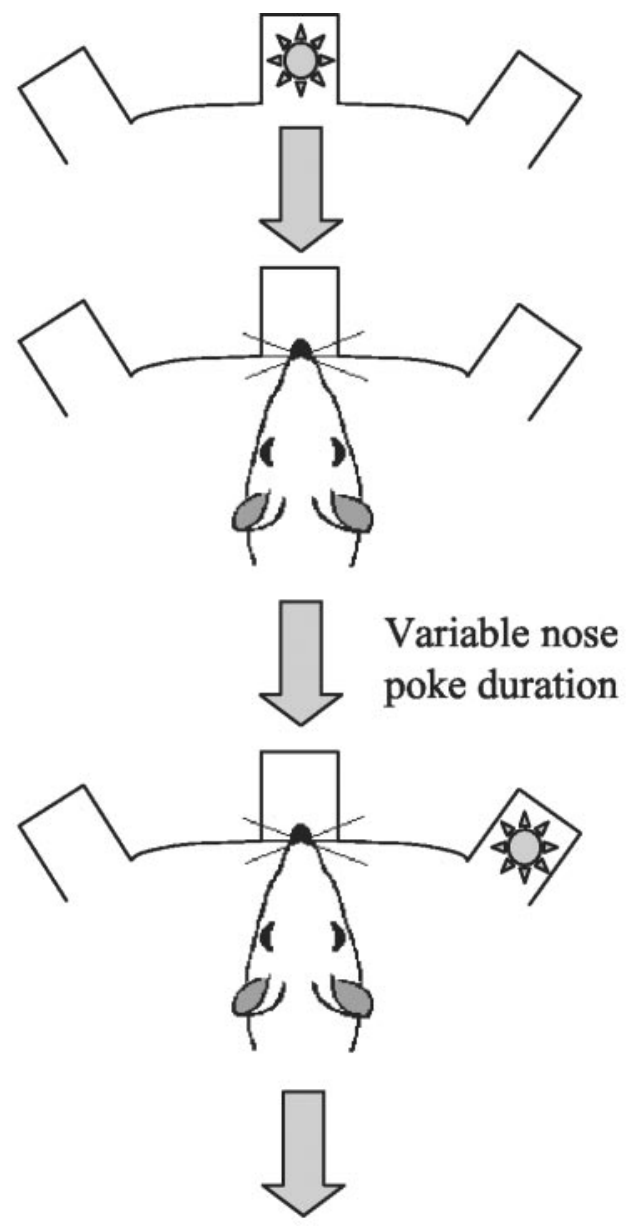

\section{Correct or incorrect choice or omission}

Figure 1. Schematic figure displaying the general task contingency. The trial onset is signaled by illumination of the central nose-poke aperture. A response of variable duration at the central aperture then results in target delivery in one of the lateral apertures. A response toward the target is reinforced; an incorrect choice or failure to respond (omission) is punished with a 3 sec time-out.

tation or the limited hold period, the animal received a $3 \mathrm{sec}$ time-out in darkness and an omission was recorded. In both cases, the time-out period was immediately followed by illumination of the house light diffuser and by the illumination of the center aperture $3 \mathrm{sec}$ later.

An additional contingency was in place to discourage premature responses. If a rat responded to either of the possible target locations before completing the sustained nose-poke (and before the target presentation), a 3 sec time-out was given (as above), and an "anticipatory response" was scored.

Dependent measures for this task included: (1) rate of both correct and incorrect choices (\% of total trials), (2) omission rate (\% of total trials), (3) total anticipatory responses, (4) total trials initiated, (5) mean initiation latency/trial (the average interval between illumination of the center nose-poke aperture and the initiation of the observing response), (6) pellet-retrieval time (the average interval between pellet delivery and head entry into the magazine), (7) correct response times (the period between target stimulus onset and a nose-poke at the response location), and (8) receptacle approaches to the pellet magazine when no pellet was available. ANOVA (factor: genotype) was used to test for group differences for all dependent measures.

Variable target-duration condition. One day after the acquisition period, rats were tested in a session in which the duration of the target stimuli $(4.0,2.0,1.0$, or $0.5 \mathrm{sec})$ was varied randomly from trial to trial within the session. As above, a response at the target location within $5 \mathrm{sec}$ 

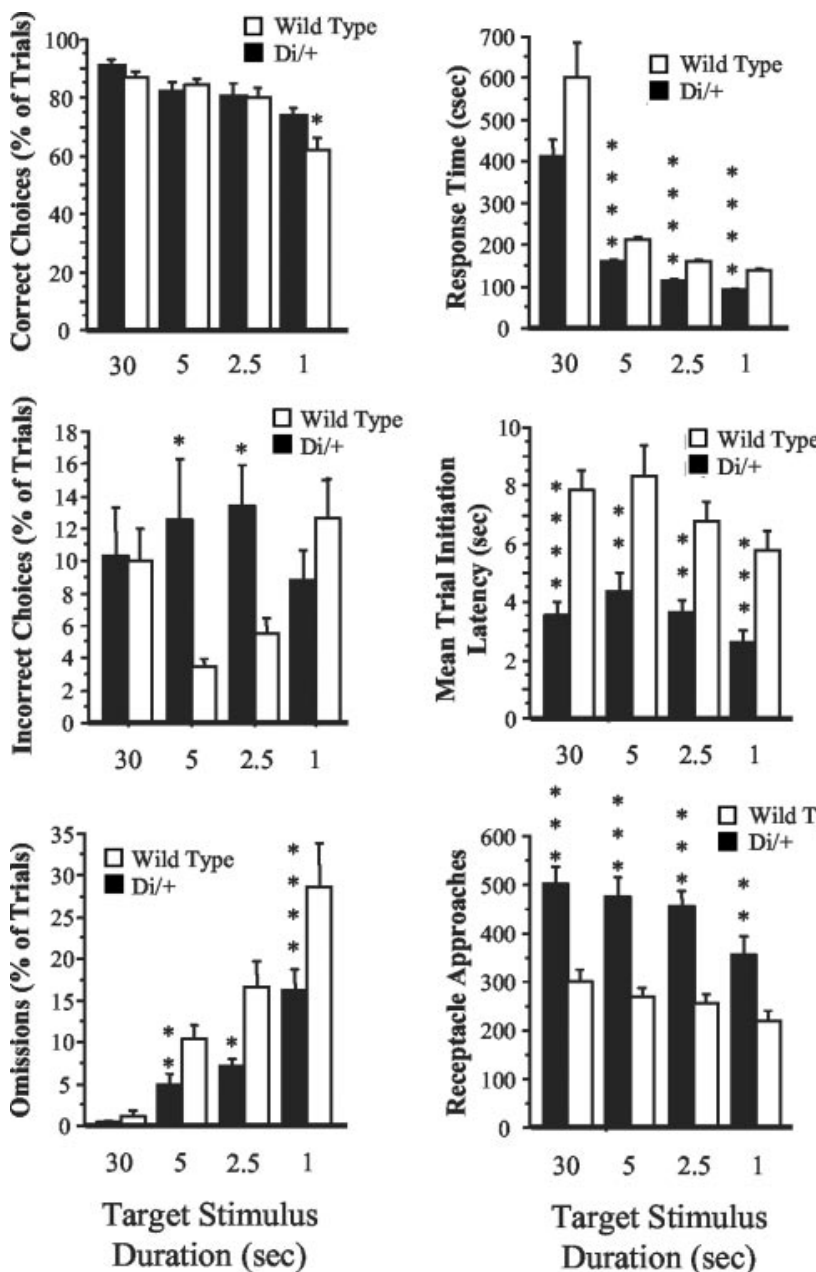

Target Stimulus Duration (sec)

Figure 2. Acquisition of the lateralized reaction-time task in di/ + Brattleboro and wildtype animals across four daily sessions in which the duration of the target stimulus was 30,5 , 2.5, or 1 sec, respectively. ${ }^{*} p<0.05 ;{ }^{* *} p<0.01 ;{ }^{* * *} p<0.001 ;{ }^{* * * *} p<0.0001$. Error bars indicate SEM.

of target onset was reinforced. All of the other task details were identical to those described above, and the dependent measures were the same, except in this case, rates of correct and incorrect choices, response times, and omission rate were analyzed with reference to the stimulus duration (repeated-measures ANOVA). Other measures were not dependent on the stimulus duration and were analyzed independently by one-way ANOVA.

Variable target-brightness condition. In a subsequent task, rats were tested in a session in which the brightness of the target stimuli $(80,60,40$, or $20 \%$ of full intensity) was varied randomly from trial to trial within the session, whereas the target duration was held constant at $1.0 \mathrm{sec}$. A response to the target location within $5 \mathrm{sec}$ of target onset was reinforced. All of the other task details were identical to those described above, and the dependent measures were the same, except here, rates of correct and incorrect choices, response times, and omission rate were analyzed with reference to stimulus brightness (repeated-measures ANOVA). Other measures were not dependent on stimulus intensity and were analyzed independently by one-way ANOVA.

\section{Results}

\section{Acquisition: The $30 \mathrm{sec}$ target}

As early as the first testing day on the target-detection task, the effects of genotype were apparent (Fig. 2). Di/ + rats were faster to initiate trials $\left(F_{(1,16)}=24.0 ; p<0.0001\right)$ and tended to show faster response times for correct responses $\left(F_{(1,16)}=4.3 ; p=\right.$

0.054). In addition, di/ + subjects made more inappropriate receptacle approaches to the pellet magazine $\left(F_{(1,16)}=22.5 ; p=\right.$ $0.0002)$. However, other measures were not affected by genotype, including correct choices $(p=0.15)$, incorrect choices $(p=$ $0.24)$, omissions $(p=0.27)$, numbers of anticipatory responses ( $p=0.47)$, and pellet-retrieval latencies $(p=0.22)$.

\section{Acquisition: The 5 sec target}

On the second day of testing (target duration, $5 \mathrm{sec}$ ) (Fig. 2), di/+ rats again showed evidence of heightened response times, including lower trial-initiation latencies $\left(F_{(1,16)}=9.2 ; p=0.008\right)$ and faster response times $\left(F_{(1,16)}=23.8 ; p=0.0002\right)$. Receptacle approaches $\left(F_{(1,16)}=17.2 ; p=0.0009\right)$ and incorrect choices $\left(F_{(1,16)}=8.1 ; p=0.01\right)$ were elevated in the $\mathrm{di} /+$ animals. Other measures were not affected by genotype, including correct choices $(p=0.82)$, numbers of anticipatory responses $(p=$ 0.83 ), or pellet-retrieval latencies $(p=0.83)$. Interestingly, despite no difference in correct choices, $\mathrm{di} /+$ subjects made dramatically fewer omission errors than did wild-type controls on this day $\left(F_{(1,16)}=9.4 ; p=0.0078\right)$, indicating a bias to choose incorrectly rather than to omit a response.

\section{Acquisition: The $2.5 \mathrm{sec}$ target}

The effects of genotype on performance were similar on day 3 , when the target duration was $2.5 \mathrm{sec}$ (Fig. 2). Trial-initiation latencies $\left(F_{(1,16)}=12.2 ; p=0.003\right)$ and correct response times $\left(F_{(1,16)}=53.2 ; p<0.0001\right)$ were again faster in $\mathrm{di} /+$ rats; receptacle approaches $\left(F_{(1,16)}=30.2 ; p<0.0001\right)$ and incorrect choices were again elevated $\left(F_{(1,16)}=5.9 ; p=0.03\right)$. Correct choices were unaffected $(p=0.75)$, and the omission rate was reduced in di/ + Brattleboro rats $\left(F_{(1,16)}=7.1 ; p=0.02\right)$. Pelletretrieval times were not affected by genotype $(p=0.17)$.

\section{Acquisition: The 1 sec target}

When the target duration was reduced to $1 \mathrm{sec}$ on day 4 (Fig. 2), an effect of genotype on correct choices became apparent $\left(F_{(1,16)}=5.8 ; p=0.02\right)$; the effect of genotype on the omission rate just failed to reach significance $(p=0.05)$. Trial-initiation latencies $\left(F_{(1,16)}=16.9 ; p=0.0008\right)$ and correct response times $\left(F_{(1,16)}=66.4 ; p<0.0001\right)$ were shorter in $\mathrm{di} /+$ rats; receptacle approaches were elevated in $\mathrm{di} /+$ rats $\left(F_{(1,16)}=10.4 ; p=0.005\right)$. Pellet-retrieval times were not affected by genotype $(p=0.17)$, nor was the number of anticipatory responses $(p=0.23)$ or incorrect choices $(p=0.92)$. Under this condition, di/ + animals showed slightly longer pellet-retrieval times, although the difference did not reach significance ( $p=0.07)$.

\section{Variable target-duration session}

Data for the variable target-duration session are shown in Figure 3. For the session in which target-stimulus durations were varied randomly $(0.5,1.0,2.0$, or $4.0 \mathrm{sec})$ from trial to trial within a session, we found a main effect of target duration for the rate of correct choices $\left(F_{(3,48)}=30.0 ; p<0.0001\right)$, incorrect choices $\left(F_{(3,48)}=8.8 ; p<0.0001\right)$, response times $\left(F_{(3,48)}=2.9 ; p=\right.$ $0.04)$, and omission rate $\left(F_{(3,48)}=12.4 ; p<0.0001\right)$; these main effects were produced by fewer correct choices and more incorrect choices and omissions at the $0.5 \mathrm{sec}$ versus the $4.0 \mathrm{sec}$ condition. There were main effects of genotype for correct choices $\left(F_{(1,16)}=4.9 ; p=0.04\right)$, response times $\left(F_{(1,16)}=24.7 ; p<\right.$ $0.0001)$, and omission rate $\left(F_{(1,16)}=4.9 ; p=0.04\right)$, but not for incorrect choices $(p=0.97)$. The main effects of genotype and target duration interacted for correct choices $\left(F_{(3,48)}=3.4 ; p=\right.$ $0.02)$. 

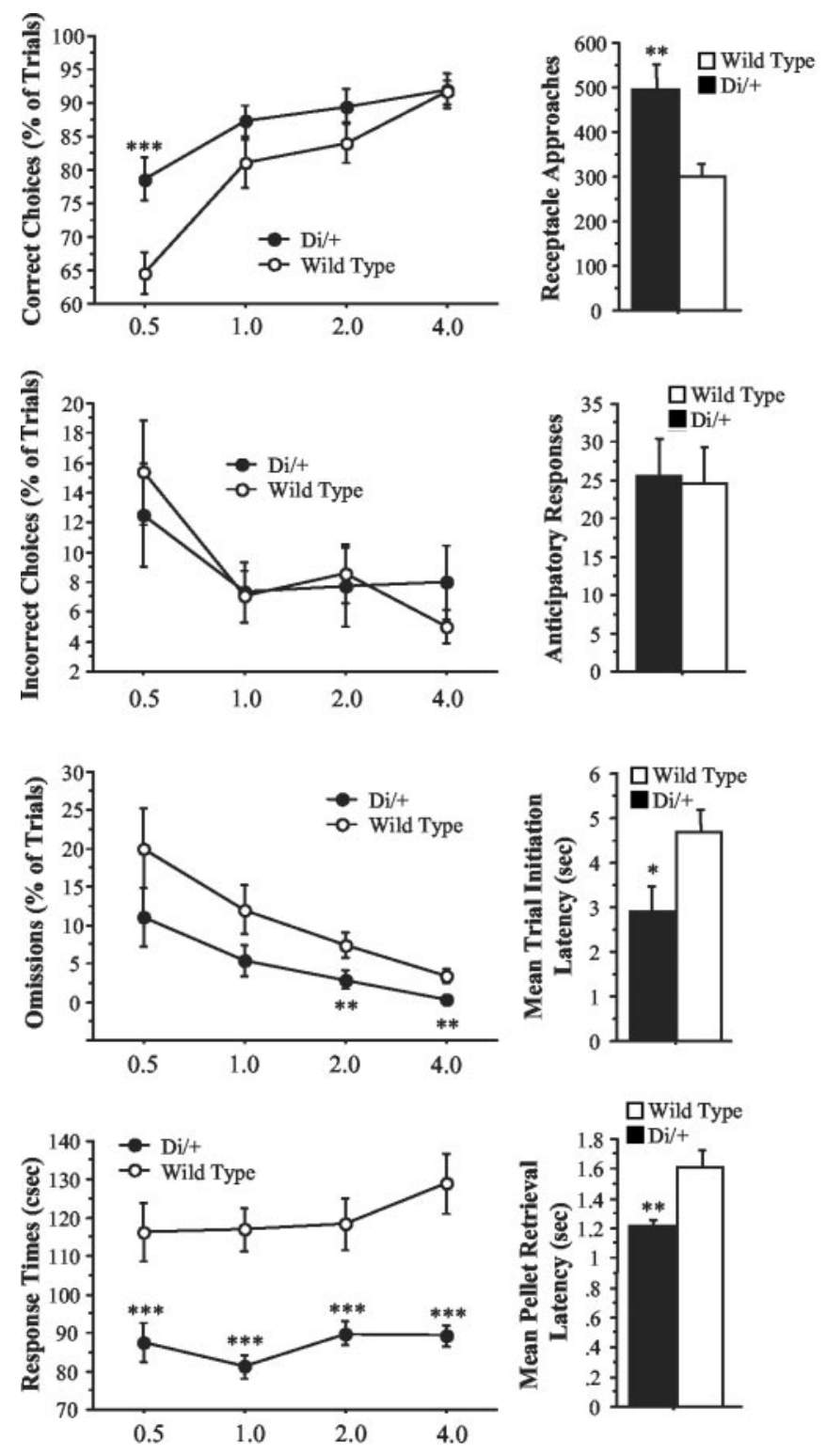

Target Stimulus Duration (sec)

Figure 3. Performance of a task condition in which target-stimulus durations are randomly varied within session. Target durations are $0.5,1.0,2.0$, or 4.0 sec. ${ }^{*} p<0.05$; ${ }^{* *} p<0.01$; ${ }^{* * *} p<0.001$. Error bars indicate SEM.

These main effects and interaction were also analyzed by post hoc comparisons. Scheffé's $F$ test revealed that the main effect of genotype and genotype $\times$ target-duration interaction were produced by a dramatic facilitation of correct choices in the di/ + rats only at the briefest duration condition $(0.5 \mathrm{sec} ; p<0.001)$. In contrast, the main effect of genotype for response times was driven by significantly faster response initiations in the di/ + rats at all target durations (all $p<0.001$ ). The main effect of genotype for omissions was driven by fewer omissions in $\mathrm{di} /+$ rats that were significant for the 2.0 and $4.0 \mathrm{sec}$ conditions only $(p<$ $0.01)$.

$\mathrm{Di} /+$ rats also showed other alterations of performance. Genotype affected the mean trial-initiation latency $\left(F_{(1,16)}=5.4\right.$; $p=0.03)$ and pellet-retrieval times $\left(F_{(1,16)}=10.1 ; p=0.005\right)$; for both measures, di/ + rats were significantly faster than their wild-
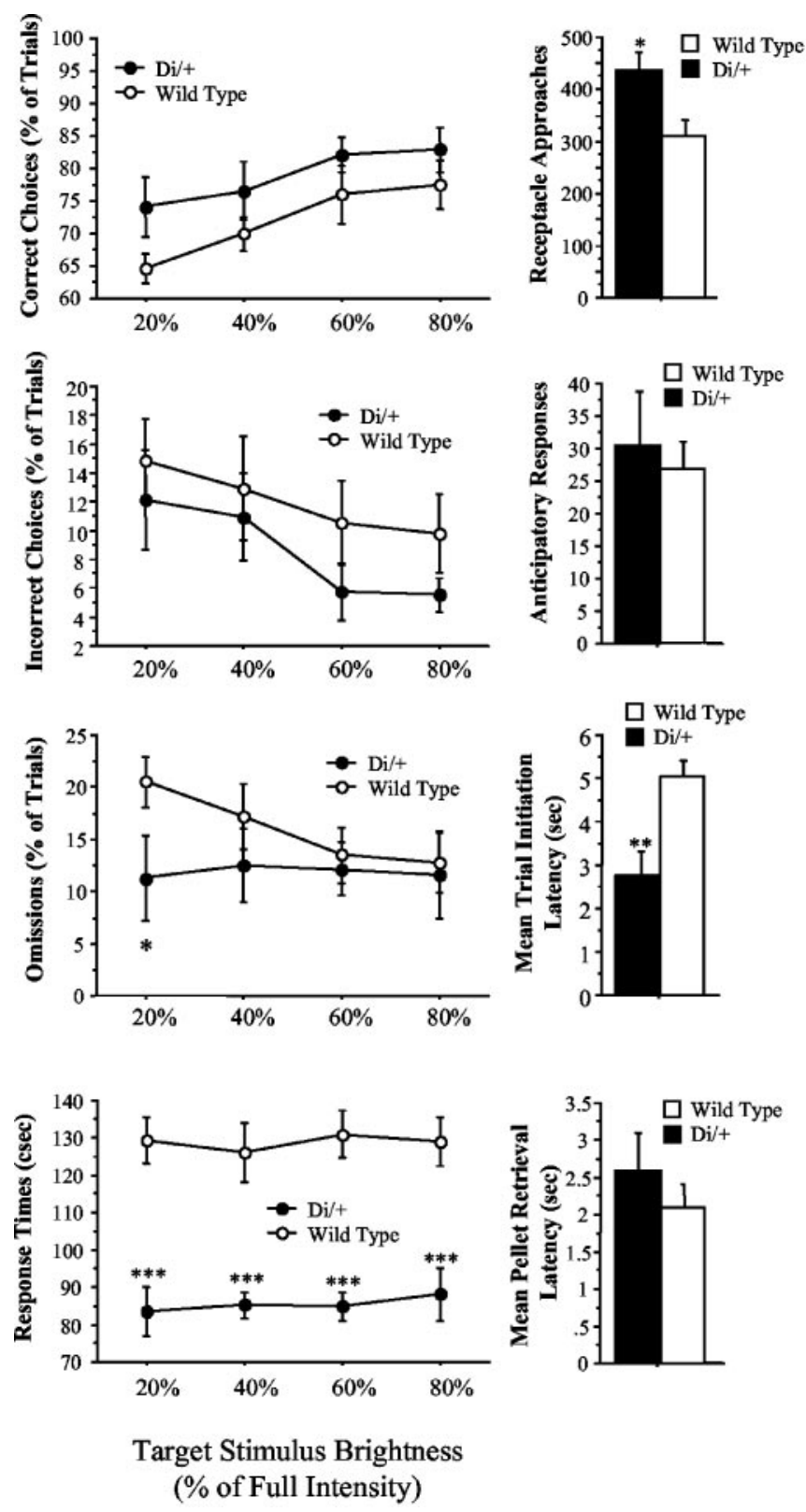

Figure 4. Performance of a task condition in which target stimulus brightness is randomly varied within session. Target brightness is $20,40,60$, or $80 \%$ of full intensity. Target duration is fixed at 1.0 sec. ${ }^{*} p<0.05 ;{ }^{* *} p<0.01 ;{ }^{* * *} p<0.001$. Error bars indicate SEM.

type counterparts. $\mathrm{Di} /+$ animals also made more approaches to the pellet receptacle $\left(F_{(1,16)}=7.8 ; p=0.02\right)$, although neither anticipatory responses nor total trials initiated were affected by genotype.

\section{Variable target-brightness session}

Data for the variable target-intensity session are shown in Figure 4. For the session in which target-stimulus brightness was varied $(20,40,60$, or $80 \%$ of full intensity) randomly from trial to trial within a session, we found a main effect of target brightness for correct choices $\left(F_{(3,48)}=5.9 ; p=0.002\right)$ and incorrect choices $\left(F_{(3,48)}=4.5 ; p=0.0068\right)$ but not for response times or omission rate. The main effect of genotype for correct choices nearly reached significance $\left(F_{(1,16)}=3.6 ; p=0.07\right)$; response times were strongly affected by genotype $\left(F_{(1,16)}=31.4 ; p<0.0001\right)$. There were no main effects of genotype for omissions $(p=0.27)$ or 
incorrect choices ( $p=0.3$ ); however, genotype interacted with target brightness for omissions $\left(F_{(1,16)}=3.2 ; p=0.03\right)$.

The main effect of genotype for response times in the variablebrightness condition was driven by significantly faster response initiations in the di/ + rats at all target intensities (all $p<0.001$ ). The genotype $\times$ target brightness interaction for omission rate was driven by higher rates of omissions in wild-type rats at only the $20 \%$ intensity condition $(p<0.05)$.

In the variable-brightness task condition, receptacle approaches were higher in $\mathrm{di} /+\operatorname{rats}\left(F_{(1,16)}=7.9 ; p=0.01\right)$. $\mathrm{Di} /+$ rats also exhibited quicker mean trial-initiation times $\left(F_{(1,16)}=\right.$ $12.1 ; p=0.003)$. No other measures, including anticipatory responses or pellet-retrieval times, were affected by genotype.

\section{Discussion}

Heterozygous Brattleboro rats that are partially vasopressindeficient exhibit superior visuospatial attention performance and faster motor-initiation times compared with wild-type LongEvans rats, as assessed by a lateralized reaction-time task. Using both between- and within-session approaches to examine the effects of variable target duration on performance, we found that di/+ Brattleboro rats were more accurate at detecting relatively brief, but not longer, visual target stimuli and showed faster motor-initiation times compared with wild-type rats. This greater probability of correct responses was associated with a specific decrease in omissions and no change in the rate of incorrect choices (in the variable duration and brightness conditions), indicating a specific facilitation of correct choices and suggesting that di/+ rats were less likely to "miss" the delivery of the target stimuli.

To determine whether motor speeding in $\mathrm{di} /+$ rats alone accounted for the choice accuracy phenotype, we examined performance in an additional session in which the brightness but not duration of visual targets was varied among trials. This condition accounts for possible differences in response speed, because in this case, the time between the nose-poke and stimulus onset and offset is held constant across trials. We found a strong trend for a main effect of genotype ( $p=0.07)$, suggesting that the phenotype is reduced but not eliminated. These results indicate that the phenotype of di/ + likely includes both facilitated attention performance and faster motor-initiation speeds.

\section{Vasopressin effects on attention and memory}

A host of studies, using both Brattleboro rats and/or administration of systemically available vasopressin analogs, has provided evidence for a role for vasopressinergic systems in memory and attention (Strupp and Levitsky, 1985; Insel et al., 1999). For example, homozygous Brattleboro rats have been reported to show deficits of working memory (Colombo et al., 1992). Perhaps paradoxically, the systemic administration of vasopressin receptor agonists also impairs short-term memory (Sahgal, 1987).

Sahgal (1988) provided evidence that the deficits of shortterm memory produced by vasopressin analogs may be attributable to a primary deficit of visual attention. This finding is roughly consistent with the current observations and would tend to indicate that vasopressinergic tone may exert a functionally deleterious influence on visual attention and/or short-term memory.

The locus of action by which vasopressin induces neurocognitive alterations is unclear. Both the systemic administration of vasopressin analogs and the genetic disruption of vasopressin synthesis are expected to alter vasopressin availability equivalently across tissues. Therefore, it remains possible that the phe- notype of di/ + rats can result from either direct alterations of vasopressinergic signaling in brain or from an indirect effect of a primary alteration within peripheral tissues. Additional studies using intracerebral infusions of vasopressinergic analogs should help to clarify this issue.

\section{Indirect alterations of monoaminergic function?}

The phenotype of di/ + rats is similar in some (but not all) aspects to that produced by systemically administered stimulants ( $d$ amphetamine or methylphenidate). Both agents, within a lowdose range, can speed responses and improve choice accuracy in tasks of visual attention (Sostek et al., 1980); however, stimulants also increase anticipatory responses (Harrison et al., 1997), a phenotype not seen in di/+ rats. However, it is possible that alterations of monoamines, secondary to changes in vasopressin signaling, could contribute, in part, to the performance differences between di/ + and wild-type rats. Of relevance, studies of homozygous Brattleboro rats have provided evidence for increased tissue contents of monoamines in a wide number of brain regions (Dawson et al., 1990; Feenstra et al., 1990). Although neither study provided direct evidence for heightened transmission of monoaminergic systems (both were measures of basal levels), it is likely that the higher transmitter reserve could result in a greater release of transmitter during metabolically demanding conditions. Therefore, it is noteworthy that the performance of visuospatial attention tasks is known to be associated with a greater release of noradrenaline within cerebral cortical regions (Dalley et al., 2001). It is possible, then, that the greater catecholamine reserve in Brattleboro rats could be invoked during cognitive performance, leading to some of the behavioral differences reported here.

\section{Relevance to neurodevelopmental disorders}

Several investigators have described the vasopressin-deficient Brattleboro rat as a possible model for schizophrenia and/or autism (Insel et al., 1999; Feifel and Priebe, 2001). Both disorders include components of altered attention resources, as well as deficient attention regulation. For example, patients with schizophrenia show impairments of sustained and divided attention as well as of attention shifting (Nuechterlein, 1977). In contrast, autism is associated with intact, or possibly superior, low-level focused attention (Plaisted et al., 1999; O'Riordan and Plaisted, 2001; O'Riordan et al., 2001) coupled with dysfunctional executive aspects of attention (Allen and Courchesne, 2001).

The current results are inconsistent with the notion that the heterozygous Brattleboro rat models the attention dysfunction reported in schizophrenia. Di/+ Brattleboro rats exhibit deficits of sensorimotor gating (Feifel and Priebe, 2001), a phenotype with a strong similarity to a primary symptom of schizophrenia. However, the current results indicate that di/ + rats do not model one of the core features of schizophrenia, namely visual attention dysfunction (Green and Nuechterlein, 1999). Clearly, it remains possible that the homozygous Brattleboro rat may be more useful in this regard, and additional studies should be directed toward investigating this issue.

The potential contribution of the $\mathrm{di} /+$ Brattleboro rat for autism research warrants additional investigation. Enhanced visual search and discrimination in autism (Plaisted et al., 1999; O'Riordan and Plaisted, 2001; O'Riordan et al., 2001) may result from facilitations of low-level attention; therefore, di/+ Brattleboro rats may mimic that feature of the idiopathic disease. However, the confirmation of this relationship awaits additional studies of shifting attention and/or executive aspects of attention 
control in $\mathrm{di} /+$ rats, processes that should be impaired in a valid animal model for autism.

\section{Conclusions}

Rats with a genetic deficiency in vasopressin signaling show a clear phenotype of enhanced visuospatial attention performance and faster motor-initiation times. The primary role for decreased vasopressin release and/or the secondary role for monoaminergic alterations deserve additional study. These studies may ultimately clarify the relevance of alterations of neurophysin signaling in neurodevelopmental disorders.

\section{References}

Allen G, Courchesne E (2001) Attention function and dysfunction in autism. Front Biosci 6:D105-D119.

Barberis C, Tribollet E (1996) Vasopressin and oxytocin receptors in the central nervous system. Crit Rev Neurobiol 10:119-154.

Carli M, Robbins TW, Evenden JL, Everitt BJ (1983) Effects of lesions to ascending noradrenergic neurones on performance of a 5-choice serial reaction task in rats:implications for theories of dorsal noradrenergic bundle function based on selective attention and arousal. Behav Brain Res 9:361-380.

Colombo G, Hansen C, Hoffman PL, Grant KA (1992) Decreased performance in a delayed alternation task by rats genetically deficient in vasopressin. Physiol Behav 52:827-830.

Dalley JW, McGaughy J, O'Connell MT, Cardinal RN, Levita L, Robbins TW (2001) Distinct changes in cortical acetylcholine and noradrenaline efflux during contingent and noncontingent performance of a visual attentional task. J Neurosci 21:4908-4914.

Dawson Jr R, Wallace DR, King MJ (1990) Monoamine and amino acid content in brain regions of Brattleboro rats. Neurochem Res 15:755-761.

Engelmann M, Landgraf R (1994) Microdialysis administration of vasopressin into the septum improves social recognition in Brattleboro rats. Physiol Behav 55:145-149.

Feenstra MG, Snijdewint FG, Van Galen H, Boer GJ (1990) Widespread alterations in central noradrenaline, dopamine, and serotonin systems in the Brattleboro rat not related to the local absence of vasopressin. Neurochem Res 15:283-288.

Feifel D, Priebe K (2001) Vasopressin-deficient rats exhibit sensorimotor gating deficits that are reversed by subchronic haloperidol. Biol Psychiatry 50:425-433.

Green MF, Nuechterlein KH (1999) Should schizophrenia be treated as a neurocognitive disorder? Schizophr Bull 25:309-319.

Harrison AA, Everitt BJ, Robbins TW (1997) Central 5-HT depletion enhances impulsive responding without affecting the accuracy of attentional performance: interactions with dopaminergic mechanisms. Psychopharmacology (Berl) 133:329-342.

Insel TR, O'Brien DJ, Leckman JF (1999) Oxytocin, vasopressin, and autism: is there a connection? Biol Psychiatry 45:145-157.

Nuechterlein KH (1977) Refocusing on attentional dysfunctions in schizophrenia. Schizophr Bull 3:457-.

O’Riordan M, Plaisted K (2001) Enhanced discrimination in autism. Q J Exp Psychol A 54:961-979.

O'Riordan MA, Plaisted KC, Driver J, Baron-Cohen S (2001) Superior visual search in autism. J Exp Psychol Hum Percept Perform 27:719-730.

Pantelis C, Barnes TR, Nelson HE, Tanner S, Weatherley L, Owen AM, Robbins TW (1997) Frontal-striatal cognitive deficits in patients with chronic schizophrenia. Brain 120:1823-1843.

Plaisted K, Swettenham J, Rees L (1999) Children with autism show local precedence in a divided attention task and global precedence in a selective attention task. J Child Psychol Psychiatry 40:733-742.

Sahgal A (1987) Contrasting effects of vasopressin, desglycinamidevasopressin,and amphetamine on a delayed matching to position task in rats. Psychopharmacology (Berl) 93:243-249.

Sahgal A (1988) Vasopressin and amphetamine, but not desglycinamide vasopressin, impair positively reinforced visual attention performance in rats. Behav Brain Res 29:35-42.

Sostek AJ, Buchsbaum MS, Rapoport JL (1980) Effects of amphetamine on vigilance performance in normal and hyperactive children. J Abnorm Child Psychol 8:491-500.

Stoehr JD, Cheng SW, North WG (1993) Homozygous Brattleboro rats display attenuated conditioned freezing responses. Neurosci Lett 153:103-106.

Strupp BJ, Levitsky DA (1985) A mnemonic role for vasopressin: the evidence for and against. Neurosci Biobehav Rev 9:399-411. 